\title{
Treatment of Irritability in Huntington's Disease
}

\section{Erik van Duijn, MD, PhD}

\author{
Address \\ GGZ Delfland, P0 Box 5016, 2600 GA, Delft, The Netherlands \\ Email: e.vanduijn@ggz-delfland.nl \\ Published online: 10 July 2010 \\ (C) The Author(s) 2010. This article is published with open access at Springerlink.com
}

\section{Opinion statement}

Irritability is a common neuropsychiatric feature of Huntington's disease (HD), with prevalences varying from $38 \%$ to $73 \%$. Similar prevalences of irritability are reported in other neurodegenerative disorders and traumatic brain injury, especially when the frontal lobe is involved. Before therapeutic interventions are initiated, the clinician should analyze the severity and frequency of the irritable behavior. By examining irritability in a broader spectrum, a tailor-made treatment can be provided.

In general, I recommend as a first step a selective serotonin reuptake inhibitor (SSRI), such as sertraline, or the mood stabilizer valproate; they both have a mild side effect profile. Next, if the result is insufficient, I advise a switch between these two medications. As an alternative, I recommend a switch to a low dose of an atypical antipsychotic, preferably twice daily. Buspirone may be another alternative. Both antipsychotics and buspirone are also used as an add-on. Other mood stabilizers and beta-adrenergic receptor antagonists should only be used when earlier treatments are ineffective. The use of acetylcholinesterase inhibitors for the treatment of irritability is discouraged, as results are unclear. Synthetic cannabinoids are an interesting new therapeutic option, though their "illicit" compound and side effect profile make them not a first-line option.

It is important to identify possible comorbid psychiatric disorders, because irritability may be secondary to a psychiatric condition, and the choice of medication partly depends on the co-occurrence of a specific psychiatric disorder. For example, antipsychotic medication would be the treatment of choice in delusional HD patients with excessive irritability, instead of an SSRI or valproate.

Besides psychiatric comorbidity, the choice of medication also depends on the general medical condition, the side effect profile, and drug-drug interactions with other medications in concomitant use. Patients with advanced disease are particularly likely to be using various other types of medications.

In addition to pharmacotherapy, behavioral therapy or other psychotherapeutic interventions may be helpful to reduce levels of stress and should be considered. 


\section{Introduction}

Huntington's disease (HD) is a progressive neurodegenerative disorder characterized by motor symptoms, cognitive decline, and psychiatric disorders [1]. HD is caused by a trinucleotide expansion in the IT15 gene, coding for the mutant protein huntingtin, and has an autosomal dominant hereditary pattern. Neurodegeneration occurs primarily in the striatum and cerebral cortex. The onset of HD most commonly occurs between 30 and 50 years of age, and the average disease duration is 16 years.

Although motor symptoms remain at the forefront of the clinical diagnosis, neuropsychiatric symptoms often precede the onset of motor symptoms. Next to depression, obsessive-compulsive disorder, and anxiety, irritability and aggression are frequent neuropsychiatric symptoms in HD $[2,3,4]$. Irritable patients are frequently hard to get along with, have emotional lability and eruptions, and demonstrate outbursts in response to minor provocations. The burden of this neuropsychiatric symptom is highly associated with functional disability.

The term irritability is often poorly defined and is used as synonym for agitation, hostility, aggressive behavior, and violent outbursts. Irritability is best defined as a temporary mood state characterized by impatience, intolerance, and reduced control over temper, which usually results in verbal or behavioral outbursts. It includes elements of anger, aggression, and reduced impulse control and can occur independently of other neuropsychiatric conditions $[5,6]$.

A variety of psychotropic medications are used to treat irritability in HD, although no medication is officially approved for this indication. In this review of the literature, we aimed to investigate all reported treatments for irritability in HD.

The present level of evidence is based on small studies, with different definitions of irritability and measurement tools, and many patients used concomitant medications that may have affected the clinical outcome. In addition to these methodologic shortcomings, the choice of the medications in the studies examined seems to be rather arbitrary. For example, only one publication is available on the use of a selective serotonin reuptake inhibitor (SSRI) [7, Class IV], whereas in clinical practice, SSRIs are often prescribed as a first-choice treatment for irritability.

Most of the studies discuss the use of antipsychotics, in particular olanzapine $[8,9,10,11$, Class IV]. Relatively large case series show an improvement of irritability after initiating treatment with olanzapine, indicating that olanzapine may be an effective treatment for irritability. However, olanzapine has not been compared with other medications, so the results are inconclusive.

So far, only one study comparing the effect of two different medications (lithium carbonate and haloperidol, in a crossover study), and their combination, has been published [12, Class III]. Recently, a placebo-controlled study measuring the effect of nabilone was published, showing an overall improvement in behavior [13•, Class III].

In conclusion, there is a low level of evidence for current treatments of irritability, and systematic research with randomized controlled trials is warranted to measure the effect of medication.

\section{Treatment}

\section{Pharmacologic treatment}

\section{Selective serotonin reuptake inhibitors (SSRIs)}

\section{Sertraline}

A hospitalized patient showed a "dramatic improvement" in irritability shortly after starting sertraline (100 $\mathrm{mg})$, in combination with an ongoing treatment of haloperidol, whereas earlier treatment with carbamazepine was not effective [7, Class IV]. Another patient improved with sertraline after treatments with several other psychotropic medications had been ineffective [7, Class IV].

Contraindications No strict contraindications. 
Main drug interactions

Main side effects

Special points
Sertraline should not be used in combination with monoamine oxidase inhibitors or with the antipsychotic drug pimozide. Sertraline may displace warfarin from plasma proteins and increase the prothrombin time. Because sertraline is metabolized by the hepatic CYP2D6 enzyme, sertraline may interfere with the metabolism of other drugs that are metabolized by CYP2D6, especially in so-called poor metabolizers [14].

Most adverse effects appear within the first 1 to 2 weeks, and they generally subside or resolve spontaneously. Sexual dysfunction, gastrointestinal problems (nausea, diarrhea, anorexia, vomiting, and dyspepsia), weight gain or loss, and headache are common adverse effects of SSRIs. Less frequent adverse effects are anxiety, suicidal tendencies, insomnia, sedation, and vivid dreams or nightmares.

SSRIs require several weeks to produce a beneficial effect on behavioral problems, whereas a fast response is usually needed. SSRIs may be especially effective for irritability associated with depression.

\section{Azapirones}

\section{Buspirone}

Contraindications Buspirone should be used with caution by persons with hepatic or renal impairment.

Main drug interactions If buspirone is used in combination with serotonergic agents, there is an increased risk for the occurrence of a serotonin syndrome. Buspirone may increase the plasma concentration of haloperidol [14]. Agents with an inhibitory effect on CYP3A4 (eg, diltiazem, verapamil, itraconazole, erythromycin) increase the buspirone plasma concentration.

Main side effects The most common adverse effects of buspirone are headache, nausea, dizziness, and sometimes feelings of restlessness. Buspirone has no sedative or hypnotic effects.

Special points The short half-life (2 to 11 hours) necessitates dosing three times daily.

\section{Atypical neuroleptics}

\section{Olanzapine}

In an open-label study, $11 \mathrm{HD}$ patients were treated with olanzapine for several indications, including disruptive behavior and "psychomotor agitation" [11, Class IV]. After 6 and 12 months, the behavioral score of the Unified Huntington's Disease Rating Scale (UHDRS) [18] was significantly reduced, whereas the UHDRS motor score was unchanged. 
Contraindications

Main drug interactions

Main side effects

Special points
Olanzapine (5 mg) was a useful antipsychotic drug in an open-label study with 10 patients, with significant changes in behavior, including irritability [8, Class IV]. Reported dosages of olanzapine as a treatment for agitation vary between $5 \mathrm{mg}$ and $10 \mathrm{mg}$ daily in combination with valproate [9, Class IV]. Impulsivity and aggressive behavior diminished with $5 \mathrm{mg}$ of olanzapine. Chorea movements and functioning in activities of daily life improved when the dose was increased to $10 \mathrm{mg}$ per day [10, Class IV].

Risk for narrow-angle glaucoma.

Concentrations of olanzapine are increased by coadministration of the CYP1A2 inhibitor fluvoxamine and decreased by coadministration of the CYP1A2 inducer carbamazepine.

Somnolence, orthostatic hypotension, hyperglycemia, dry mouth, constipation, dyspepsia, increased appetite, prolonged QT interval, and tremor are associated with olanzapine use. Olanzapine is more likely than the other neuroleptics to cause weight gain.

The dopamine $\mathrm{D}_{2}$ antagonist properties of olanzapine may explain its possible benefits in the improvement of chorea.
Contraindications

Main drug interactions

Main side effects
In a patient with anger, anxiety, and impulsive behavior, risperidone was initiated after a treatment with haloperidol. The dosage was titrated gradually up to $1 \mathrm{mg}$ twice daily, giving a moderate improvement in movement and overall psychiatric symptoms [19, Class IV]. Another case report described a late-onset HD patient who showed behavioral changes with temper outbursts and psychotic symptoms, which disappeared after starting risperidone at a dosage of $1 \mathrm{mg}$ twice daily [20, Class IV]. A retrospective review of a group of HD patients treated with risperidone (average dose, $2.5 \mathrm{mg}$ ) for an average of 15 months showed improved psychiatric functioning measured with the UHDRS behavioral score, but the effect on irritability was not specified [21•, Class III].

No strict contraindications.

Concurrent use of risperidone and phenytoin or SSRIs may produce extrapyramidal symptoms [14]. Carbamazepine has been shown to decrease the plasma concentration of risperidone. Otherwise, risperidone has little effect on other drugs.

The most frequent adverse effects observed with risperidone are insomnia, agitation, anxiety, headache, weight gain, nausea and vomiting, and extrapyramidal effects. Less frequent effects are prolonged QT interval, postural hypotension, dizziness, fatigue, sexual dysfunction, and hyperglycemia.

With dosages varying from $150 \mathrm{mg}$ to $300 \mathrm{mg}$ daily, improvement of behavioral symptoms, including irritability, was noted in five HD patients without worsening of motor functioning [22, Class IV]. However, all patients had multiple psychiatric symptoms and were treated with several types of medications. In a case report concerning an elderly HD patient with hypersexuality, the addition of quetiapine $(25 \mathrm{mg}$ at bedtime) to cyproterone for his deviant sexual behavior was effective in reducing agitation $[23$, Class IV]. 
Contraindications No strict contraindications.

Main drug interactions Coadministration of the CYP3A4 inducers carbamazepine and phenytoin increases the clearance of quetiapine. Inhibitors of CYP3A4, such as cimetidine, ketoconazole, and protease inhibitors, reduce quetiapine clearance [14].

Main side effects The most common adverse effects of quetiapine are somnolence, headache, orthostatic hypotension, and dizziness, which are usually transient. Quetiapine is also associated with weight gain, constipation, increase of heart rate, prolonged QT interval, and rise in liver transaminases.

\section{Aripiprazole}

\section{Contraindications}

Main drug interactions

Main side effects
In a patient with irritability and delusions, aripiprazole (20 mg per day) gave an improvement of his psychotic symptoms and his personal hygiene, though the effect on irritability remained unclear [24, Class IV]. One advanced symptomatic patient with agitation showed an improvement of agitation with aripiprazole (15 mg per day), with good control of motor symptoms and without adverse effects [25, Class IV]. However, precise assessment of behavioral functioning in this patient was not possible because of severe cognitive dysfunction.

No strict contraindications.

Coadministration of the CYP3A4 inducers carbamazepine and phenytoin increases the clearance of aripiprazole. Inhibitors of CYP3A4, such as cimetidine, ketoconazole, and protease inhibitors, reduce aripiprazole clearance. CYP2D6 inhibitors (eg, quinidine) increase aripiprazole plasma concentrations [14].

Orthostatic hypotension, nausea, and vomiting are the most frequent adverse effects. Aripiprazole rarely induces extrapyramidal and metabolic adverse effects.

\section{Mood stabilizers}

\section{Valproate}

One study reported an effective treatment of agitation with valproate, $1500 \mathrm{mg}$ daily, with a plasma concentration range from 60 to $80 \mu \mathrm{g} / \mathrm{mL}$, in combination with olanzapine [19, Class IV].

Contraindications Preexisting hepatic or renal failure, hepatitis, pancreatitis, bone marrow depression, and hematologic coagulation disorders are contraindications.

Main drug interactions Coadministration with lithium may result in a drug-induced tremor. Valproate also interacts with carbamazepine. Valproate decreases the clearance of amitriptyline and nortriptyline [14].

Main side effects Nausea, vomiting, dyspepsia, and diarrhea are the most common adverse effects in the first month of treatment. Other possible effects are sedation, ataxia, dysarthria, tremor, hair loss, and weight gain.

Carbamazepine $(500 \mathrm{mg} / \mathrm{d})$ was effectively used in combination with haloperidol, clonazepam, and a strict behavior modification plan in a patient with aggressive and disinhibited behavior [26, Class IV].

Contraindications Atrioventricular block, a history of bone marrow depression, acute porphyria, and hepatic or renal failure are relative contraindications. 
Main drug interactions

Main side effects

Because carbamazepine induces several hepatic enzymes, it may interact with many drugs. For the most part, adding carbamazepine lowers the plasma concentration of affected drugs, including oral contraceptives and warfarin. Carbamazepine may increase plasma concentrations of clomipramine and phenytoin. Carbamazepine should not be used in combination with monoamine oxidase inhibitors.

Adverse effects particularly occur in the initial stages of therapy. These effects include drowsiness, sedation, headache, ataxia, nystagmus, diplopia, cardiac conduction disorder, dermatologic problems, and hepatitis. Blood dyscrasia is a severe, but rare adverse effect of carbamazepine.

\section{Lamotrigine}

Contraindications

Main drug interactions

Main side effects

Contraindications

Main drug interactions

Main side effects
Only one study describes an effective treatment with lamotrigine $(300 \mathrm{mg} / \mathrm{d})$, together with clonazepam $(4 \mathrm{mg} / \mathrm{d})$ in a patient with irritability, mood swings, and depression [27, Class IV].

No strict contraindications.

The lamotrigine concentration is increased with concurrent administration of valproate, and to a lesser extent with sertraline. Lamotrigine plasma concentrations are decreased with concomitant administration of carbamazepine, phenytoin, or phenobarbital [14].

Common adverse effects are dizziness, ataxia, somnolence, headache, diplopia, blurred vision, nausea, vomiting, and dermatologic side effects. These dermatologic problems vary from self-limiting skin rashes to severe StevensJohnson syndrome.

\section{Lithium carbonate}

In a small, double-blind, crossover trial with six female patients, a combination of lithium and haloperidol during 3 weeks resulted in a decrease of irritability and angry outbursts, but this effect did not occur when either drug was administered separately [12, Class III].

Renal failure, cardiac conduction disorder, and Addison's disease are contraindications. Lithium should be used with caution in patients with diabetes mellitus and hypothyroidism.

Coadministration of lithium with anticonvulsants or antipsychotics may increase lithium concentrations and aggravate adverse effects. Most diuretics decrease renal lithium clearance and increase lithium concentrations. Nonsteroidal anti-inflammatory drugs can decrease lithium clearance, thereby increasing lithium concentrations. Combining lithium with methyldopa, verapamil, or diltiazem may cause neurotoxicity [14].

The most common adverse effects of lithium are increased thirst, polyuria, edema, tremor, thyroid dysfunction, weight gain, fatigue, diarrhea, nausea, vomiting, cardiac effects, dermatologic problems, and mild cognitive impairment.

\section{Acetylcholinesterase inhibitors}

No clinical or statistical improvement in behavioral, cognitive, or functional assessment was measured in an open-label study with $10 \mathrm{mg}$ per 
Contraindications

Main drug interactions

Main side effects

Special points

day of donepezil in eight symptomatic HD patients [28, Class IV]. However, a high dropout rate of 50\% was noted after 6 weeks of treatment. No strict contraindications.

Metabolism of donepezil may be increased by phenytoin, carbamazepine, rifampicin, or phenobarbital.

Nausea, diarrhea, anxiety, sedation, fatigue, vomiting, and weight loss due to anorexia are possible adverse effects of donepezil. Donepezil has been related to bradyarrhythmias.

Donepezil may have a positive effect on memory and concentration impairments.

\section{Rivastigmine}

Contraindications

Main drug interactions

Main side effects

Special points
Data on the effect of rivastigmine ( $3 \mathrm{mg}$ twice daily) show a tendency to improvement of the total score of the UHDRS behavioral scale [29, Class IV]. Severe hepatic failure.

Rivastigmine has no significant known drug interactions.

The most common adverse effects are nausea, vomiting, dizziness, headache, diarrhea, fatigue, somnolence, and anorexia.

Rivastigmine may have a positive effect on memory and concentration impairments.

\section{Beta-adrenergic receptor antagonists (beta-blockers)}

\section{Propranolol}

With dosage varying from 30 to $240 \mathrm{mg}$ daily, propranolol was an effective treatment for aggression in three patients [30, Class IV], but propranolol also precipitated paradoxic aggression in a patient with HD [31, Class IV].

Contraindications Beta-adrenergic receptor antagonists are contraindicated in people with asthma, insulin-dependent diabetes, congestive heart failure, significant vascular disease, atrioventricular conduction defect, persistent angina, or hypothyroidism.

Main drug interactions Concomitant administration of propranolol results in increased plasma concentrations of antipsychotics, anticonvulsants, theophylline, and levothyroxine [14].

Main side effects Hypotension and bradycardia are adverse effects of beta-blockers.

Special points The dosage of beta-blockers must be individually titrated, with regular measurement of pulse and blood pressure.

\section{Cannabinoids}

\section{Nabilone}

One HD patient had an improvement of mood, was calmer and more relaxed after she started smoking of cannabis [32, Class IV]. Nabilone, a synthetic 9-keto cannabinoid, was given in a larger study, resulting in an 
Contraindications Allergy to cannabinoids, history of psychosis, and liver dysfunction are contraindications for the use of nabilone.

Main drug interactions Concomitant use of benzodiazepines may potentiate the central nervous system effects of nabilone.

Main side effects Sedation, drowsiness, dizziness, forgetfulness, headache, mood changes, hallucinations, dry mouth, blurred vision, and loss of appetite are possible adverse effects of nabilone.

Special points Nabilone is available in a few countries and is indicated for treatment of nausea and vomiting associated with cancer. Theoretically, nabilone has a high potential for abuse.

\section{Other interventions}

\section{Behavioral therapy}

Both classical and operant conditioning are useful in managing irritability. Operant conditioning involves the reinforcement of more appropriate behaviors and discouragement of inappropriate behaviors [33]. Furthermore, limits and consequences of deviant behavior need to be clearly defined to the patient [34]. Especially for patients in an advanced disease stage, a behavior modification plan may include a structured daily schedule and reinforcement of desired behaviors [26].

Stressful psychosocial factors may result in irritability. After identification of stressors, the patient should be helped to look for ways to avoid these stressors and to develop concrete ways of coping, in order to decrease the level of stress within the nursing home or day care setting. Patients may be advised to break tasks down into simpler components.

Adaptive devices and specialized equipment are valuable in maintaining comfort, thereby decreasing the expression of problematic behaviors [33].

\section{Disclosure}

No potential conflicts of interest relevant to this article were reported.

\section{Open Access}

This article is distributed under the terms of the Creative Commons Attribution Noncommercial License which permits any noncommercial use, distribution, and reproduction in any medium, provided the original author(s) and source are credited. 


\section{References and Recommended Reading}

Papers of particular interest, published recently, have been highlighted as:

- Of importance

•• Of major importance

1. Walker FO: Huntington's disease. Lancet 2007, 369:218-223.

2. Kirkwood SC, Siemers E, Viken R, et al.: Longitudinal personality changes among presymptomatic Huntington's disease gene carriers. Neuropsychiatry Neuropsychol Behav Neurol 2002, 15:192-197.

3. Kingma EM, van Duijn E, Timman R, et al.: Behavioural problems in Huntington's disease using the Problem Behaviours Assessment. Gen Hosp Psychiatry 2008, 30:155-161.

4. Tabrizi SJ, Langbehn DR, Leavitt BR, et al.: Biological and clinical manifestations of Huntington's disease in the longitudinal TRACK-HD study: cross-sectional analysis of baseline data. Lancet Neurol 2009, 8:791-801.

5. Snaith RP, Taylor CM: Irritability: definition, assessment and associated factors. $\mathrm{Br} J$ Psychiatry 1985, 147:127-136.

6. Klöppel S, Stonnington CM, Petrovic P, et al.: Irritability in pre-clinical Huntington's disease. $\mathrm{Neu}$ ropsychologia 2010, 48:549-557.

7. Ranen NG, Lipsey JR, Treisman G, Ross CA: Sertraline in the treatment of severe aggressiveness in Huntington's disease. J Neuropsychiatry 1996, 8:338-340.

8. Squitieri F, Cannella M, Piorcellini A, et al.: Shortterm effects of olanzapine in Huntington's disease. Neuropsychiatry Neuropsychol Behav Neurol 2001, 14:69-72.

9. Grove VE, Quintanilla J: Improvement of Huntington's disease with olanzapine and valproate. $N$ Engl $J$ Med 2000, 343:973-974.

10. Bogelman G, Hirschmann S, Modai I: Olanzapine and Huntington's disease. J Clin Psychopharmacol 2001, 21:245-246.

11. Paleacu D, Anca M, Giladi N: Olanzapine in Huntington's disease. Acta Neurol Scand 2002, 105:441-444.

12. Leonard DP, Kidson MA, Shannon PJ, Brown J: Letter: Double-blind trial of lithium carbonate and haloperidol in Huntington's chorea. Lancet 1974, 2 (7890):1208-1209.

13. Curtis A, Mitchell I, Patel S, et al.: A pilot study using nabilone for symptomatic treatment in Huntington's disease. Mov Disord 2009, 24:2254-2259.

This interesting study used the synthetic cannabinoid nabilone in Huntington's disease.

14. Kaplan BJ, Sadock VA, Sussman N: Pocket handbook of psychiatric drug treatment, edn 4th. Philadelphia: Lippincott Williams \& Wilkins; 2006.
15. Findling RL: Treatment of aggression in juvenileonset Huntington's disease with buspirone. Psychosomatics 1993, 34:460-461.

16. Bhandary AN, Masand PS: Buspirone in the management of disruptive behaviors due to Huntington's disease and other neurological disorders. Psychosomatics 1997, 38:389-391.

17. Byrne A, Martin W, Hnatko G: Beneficial effects of buspirone therapy in Huntington's disease. $A m J$ Psychiatry 1994, 151:1097.

18. Huntington Study Group: Unified Huntington's Disease Rating Scale: reliability and consistency. Mov Disord 1996, 11:136-142.

19. Erdemoglu AK, Boratav C: Risperidone in chorea and psychosis of Huntington's disease. Eur J Neurol 2002, 9:182-183.

20. Caserta MT, Sullivan E: Late-onset Huntington's disease masquerading as normal pressure hydrocephalus. J Neuropsychiatry Clin Neurosci 2009, 21:97-98.

21. Duff K, Beglinger LJ, O'Rourke ME, et al.: Risperidone and the treatment of psychiatric, motor, and cognitive symptoms in Huntington's disease. Ann Clin Psychiatry 2008, 20:1-3.

This is a well-designed study on the effect of risperidone in Huntington's disease.

22. Alpay M, Koroshetz WJ: Quetiapine in the treatment of behavioral disturbances in patients with Huntington's disease. Psychosomatics 2006, 47:70-72.

23. Tavares A, Volpe FM: Cyproterone for treatment of hypersexuality in an elderly Huntington's disease patient. Prog NeuropsychopharmacolBiol Psychiatry 2008, 32:1994-1995.

24. Lin W-C, Chou Y-H: Aripiprazole effects on psychosis and chorea in a patient with Huntington's disease. Am J Psychiatry 2008, 165:1207-1208.

25. Ciammola A, Sassone J, Colciago C, et al.: Aripiprazole in the treatment of Huntington's disease: a case series. Neuropsychiatr Dis Treat 2009, 5:1-4.

26. Blass DM, Steinberg M, Leroi I, Lyketsos CG: Successful multimodality treatment of severe behavioral disturbance in a patient with advanced Huntington's disease. Am J Psychiatry 2001, 158:1966-1972.

27. Shen YC: Lamotrigine in motor and mood symptoms of Huntington's disease. World J Biol Psychiatry 2008, 9:147-149.

28. Fernandez HH, Friedman JH, Grace J, Beason-Hazen S: Donepezil for Huntington's disease. Mov Disord 2000, 15:173-176. 
29. Rot U, Kobal J, Sever A, et al.: Rivastigmine in the treatment of Huntington's disease [letter]. Eur J Neurol 2002, 9:689-690.

30. Stewart JT, Mounts ML, Clark Jr RL: Aggressive behavior in Huntington's disease: treatment with propranolol. J Clin Psychiatry 1987, 48:106-108.

31. Stewart JT: Paradoxical aggressive effect of propranolol in a patient with Huntington's disease. $J$ Clin Psychiatry 1987, 48:385-386.
32. Curtis A, Rickards H: Nabilone could treat chorea and irritability in Huntington's disease. J Neuropsychiatry Clin Neurosci 2006, 18:553-554.

33. Wood BE, Kim KK, Harpold GJ: Psychiatric management of Huntington's disease in extended care settings. Psychiatr Serv 2002, 53:703-705.

34. Hofmann N: Understanding the neuropsychiatric symptoms of Huntington's disease. J Neurosci Nurs 1999, 31:309-313. 